\title{
Check-list das Charophyceae do estado de Mato Grosso do Sul
}

\author{
Norma Catarina Bueno', Thamis Meurer ${ }^{2} \&$ Carlos Eduardo de Mattos Bicudo ${ }^{3}$
}

\begin{abstract}
'Universidade Estadual do Oeste do Paraná, Caixa Postal 711, 85814-110 Cascavel, Paraná, Brasil. normacatarina@hotmail.com 2Universidade Estadual de Maringá, 2. Bloco G-90, Av. Colombo 5790, 87020-900 Maringá Paraná, Brasil

${ }^{3}$ Instituto de Botânica, Núcleo de Pesquisa em Ecologia, Caixa Postal 68041, 04045-972 São Paulo, SP, Brasil
\end{abstract}

Recebido em 27.XI.2014

Aceito em 31.V.2016

DOI 10.21826/2446-8231201873s178

RESUMO - Foram identificadas 16 espécies dos gêneros Chara L. e Nitella C. Agardh (Characeae, Streptophyta) para o estado de Mato Grosso do Sul (19 $\left.12^{\prime} 03^{\prime \prime S}, 57^{\circ} 35^{\prime} 32^{\prime \prime} \mathrm{W}\right)$, Brasil. Foram registradas seis espécies de Chara: C. guairensis R. Bicudo, C. hydropitys Reichenbach, C. kenoyeri Howe, C. martiana Wallman, C. rusbyana Howe e C. socotrensis Nordstedt in Kuhn f. socotrensis R.D. Wood emend R.D. Wood. Foram registradas 10 espécies de Nitella e duas variedades que não típicas de suas respectivas espécies: $N$. acuminata A. Braun ex Wallman, $N$. axillaris A. Braun, N. cernua A. Braun, N. flagellifera J. Groves \& G.O. Allen, N. gollmeriana A. Braun, N. intermedia Nordst. in T.F. Allen, N. microcarpa A. Braun, N. microcarpa var. wrightii H. Groves \& J. Groves, $N$. microcarpa var. sieberi (A. Braun) J.C. van Raam, N. mucronata (A. Braun) Miquel, N. subglomerata A. Braun e N. tenuissima (Desvaux) Kützing.

Palavras-chave: Characeae, Chara, Nitella, Pantanal, taxonomia

ABSTRACT - Checklist of Capparaceae of the state of the Mato Grosso do Sul, Brazil. Sixteen species of Characeae (Chlorophyta) were identified

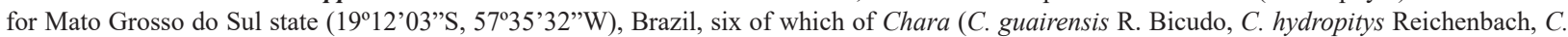
kenoyeri Howe, C. martiana Wallman, C. rusbyana Howe and C. socotrensis Nordstedt f. socotrensis) and 10 species and two varieties that are not the typical of their respective species of Nitella (N. acuminata A. Braun ex Wallman, N. axillaris A. Braun, N. cernua A. Braun, N. flagellifera J. Groves \& G.O. Allen, N. gollmeriana A. Braun, $N$. intermedia Nordstedt, $N$. microcarpa A. Braun, N. microcarpa A. Braun var. wrightii H. Groves \& J. Groves, N. microcarpa A. Braun var. sieberi (A. Braun) J.C. van Raam, N. mucronata (A. Braun) Miquel, N. subglomerata A. Braun and N. tenuissima (Desvaux) Kützing).

Keywords: Characeae, Chara, Nitella, Pantanal, taxonomy

\section{INTRODUÇÃO}

As Charophyceae constituem uma classe única de algas graças ao seu porte macroscópico e à grande complexidade morfológica tanto da organização do talo quanto da estrutura dos gametângios. No Brasil, a classe Charophyceae (família Characeae) está representada pelos gêneros Chara e Nitella, macroalgas eretas, sésseis, com eixo principal diferenciado em nós e entrenós, e com râmulos verticilados formados por filóides que sustentam os gametângios sejuntos ou conjuntos. O gênero Chara apresenta corticação no eixo principal e ápice do gametângio feminino formado por um conjunto de cinco células, enquanto o gênero Nitella apresenta eixo principal ecorticado e ápice do gametângio feminino formado por um conjunto de 10 células.

O conhecimento sobre a diversidade de espécies de Chara e Nitella no estado de Mato Grosso do Sul é escasso. Estudos apenas sobre material de Chara incluem os trabalhos de Hoehne (1914, 1923, 1936), Hoehne \&
Kuhlmann (1951), Bicudo (1968a, 1968b, 1972, 1974), Bueno et al. (1996) e Bueno et al. (2009).

Estudos sobre material de Nitella incluem os trabalhos de Braun \& Nordstedt (1883) com o registro de Nitella subglomerata A. Braun [como N. acuminata var. subglomerata (A. Braun) T. F. Allen] para Cáceres e para o rio Paraguai (Mato Grosso) e Bicudo \& Yamaoka (1978) com a citação de N. subglomerata para Brasilândia e Cuiabá (Mato Grosso). Pott et al. (1992) registraram a ocorrência de N. cernua A. Braun e N. furcata (Roxburgh ex Bruzelius) C. Agardh em lagoas do Pantanal do Abobral (Mato Grosso do Sul).

Bueno \& Bicudo (1997) identificaram seis espécies, quatro subespécies, três variedades e cinco formas taxonômicas para o Mato Grosso do Sul, sendo elas: N. acuminata A. Braun ex Wallman, N. cernua A. Braun, N. furcata (Roxburgh ex Bruzelius) C. Agardh emend R.D. Wood subsp. flagellifera (J. Groves \& G.O. Allen) R.D. Wood, N. furcata (Roxburgh ex Bruzelius) C. Agardh emend. R.D. Wood subsp. furcata var. sieberi 
(A. Braun) R.D. Wood f. microcarpa (A. Braun) R.D. Wood, N. furcata (Roxburgh ex Bruzelius) C. Agardh emend. R.D. Wood subsp. furcata var. sieberi (A. Braun) R.D. Wood, N. furcata (Roxburgh ex Bruzelius) C. Agardh emend. R.D. Wood subsp. mucronata (A. Braun) R.D. Wood var. mucronata f. mucronata, N. furcata (Roxburgh ex Bruzellius) C. Agardh emend. R.D. Wood subsp. mucronata (A. Braun) R.D. Wood var. mucronata f. wrightii (Groves \& Groves) R.D. Wood, $N$. gollmeriana A. Braun, N. subglomerata A. Braun e N. translucens (Persoon) C. Agardh emend. R.D. Wood subsp. translucens var. axillaris (A. Braun) R.D. Wood f. axillaris.

Bueno et al. (2011) identificaram nove espécies para os estados de Mato Grosso e Mato Grosso do Sul: $N$. acuminata A. Braun ex Wallman, N. axillaris A. Braun, $N$. cernua A. Braun, $N$. flagellifera J. Groves \& G.O. Allen, $N$. gollmeriana A. Braun, $N$. intermedia Nordst. in T.F. Allen, $N$. mucronata (A. Braun) Miquel in H. C. van Hall, $N$. subglomerata $\mathrm{A}$. Braun e N. tenuissima (Desvaux) Kützing.

\section{Principais grupos de pesquisa}

A Dra. Rosa Maria Teixeira Bicudo faleceu prematuramente, sem tempo para deixar descendentes. O país conta atualmente com os especialistas Dra. Maria Marcina Picelli-Vicentim, Dr. João Fernando Prado e Dra. Norma Catarina Bueno - titulados com teses sobre Characeae, dos quais os dois primeiros autores desenvolveram um trabalho taxonômico, realizando o levantamento florístico das caráceas do estado de São Paulo e Rio Grande do Sul. A terceira autora fez seu mestrado em taxonomia de carácea para os estados de Mato Grosso e Grosso do Sul. Salienta-se que a Dra. Picelli-Vicentim deixou a pesquisa há mais de 15 anos e que a Dra. Bueno hoje é a única taxonomista trabalhando com Characeae no Brasil (Universidade Estadual do Oeste do Paraná). A doutoranda Thamis Meurer (Universidade Estadual de Maringá) desenvolveu sua dissertação de mestrado "Variação da composição, distribuição espacial e influência dos fatores ambientais sobre a ocorrência de Characeae (Chlorophyta) em um reservatório subtropical", (Unioeste, Toledo) sob a orientação da Dra. Bueno e vem dando continuidade nos trabalhos de Characeae para o estado do Paraná. A mestranda Camila dos Anjos Ribeiro esta iniciando a dissertação de mestrado: "Characeae (Charophyta, Charophyceae ) das Regiões Metropolitanas de Salvador e Feira de Santana, Bahia, Brasil", na Universidade Estadual de Feira de Santana, sob a orientação do Dr. Carlos Walace do Nascimento Moura e co-orientação da Dra. Bueno e do Dr. Prado.

\section{Principais lacunas do conhecimento}

Considerando 90 espécies no mundo (Bicudo \& Bueno 2011) ou 80 espécies (Wood \& Imahori 1965), 49 no Brasil (23 espécies de Chara e 26 de Nitella) e 16 no estado do Mato Grosso do Sul, a lista de espécies de Characeae não está esgotada (Quadro 1). No Brasil existem cerca de 375 coleções depositadas nos herbários, provenientes dos países da América do Sul e Central, totalizando até o momento 60 espécies: Chara vulgaris var. vulgaris $\mathrm{f}$. vulgaris foi registrada apenas para Brasil, Peru e México; C. zeylanica var. sejunta para Argentina, Brasil e México. As espécies C. martiana Wallman, C. rusbyana Howe, $N$. clavata Kützing e $N$. golmeriana A. Braun para Argentina e Brasil. C. indica C.G. Bertero ex C. Spengel para o Brasil e Peru. C. kenoyeri Howe para Brasil e Panamá. C. canescens Desv. et Loisel in Loisel. é restrita ao México.

Para o Brasil C. hydropitys Reichenbach foi registrada até o momento para 10 estados (Bahia, Ceará, Mato Grosso, Mato Grosso do Sul, Maranhão, Minas Gerais, Paraná, Rio Grande do Sul, Sergipe e Tocantins). C. hornemanii Walmann emend. R.D. Wood por sua vez é restrita para os estados do Pernambuco e Rio de Janeiro. Nitella subglomerata A. Braun foi registrada também para 10 estados (Amazonas, Bahia, Mato Grosso, Mato Grosso do Sul, Minas Gerais, Paraná, Pernambuco, Rio de Janeiro, Rio Grande do Sul e Santa Catarina). N. acuminata A. Braun ex Wallm foi registrada para seis estados e o Distrito Federal (Goiás, Mato Grosso, Mato Grosso do Sul, Paraná, Rio de Janeiro e São Paulo). N. sieberi (A. Braun) R.D. Wood para três estados (Mato do Sul, São Paulo e Rio Grande do Sul).

Informações sobre a ocorrência de Chara no estado de Mato Grosso do Sul encontram-se nos trabalhos de Hoehne (1914, 1923, 1936), Hoehne \& Kuhlmann (1951), Bicudo (1968a, 1968b, 1972, 1974, 1976, 1977, 1979), Bueno et al. (1996) e Bueno et al. (2009). Tais informações estão de acordo com coletas realizadas na parte alta de Corumbá e em algumas "baías" do Pantanal de Aquidauna, Bodoquena, Nabileque, Nhecolândia. Os municípios que apresentaram registros de Chara foram: Aquidauna, Bodoquena, Bonito, Campo Grande, Corumbá, Inocência, Ladário e Porto Murtinho.

Até o momento foram registradas para o estado de Mato Grosso do Sul seis espécies: Chara guairensis R. Bicudo, C. hydropitys Reichenbach, C. kenoyeri Howe, C. martiana Wallman, C. rusbyana Howe e C. socotrensis Nordstedt in Kuhn f. socotrensis R.D. Wood emend R.D. Wood. Chara guairensis foi a espécie que apresentou a maior distribuição geográfica na área estudada (Aquidauna, Bodoquena, Bonito, Campo Grande, Corumbá, Inocência, Miranda, Porto Murtinho) enquanto que $C$. kenoyeri Howe e $C$. socotrensis Nordstedt foram as que apresentaram a menor distribuição, ocorrendo em apenas duas localidades cada uma (Bonito e Campo Grande e Bonito e Porto Murtinho, respectivamente). C. socotrensis Nordstedt até o momento foi registrada apenas para os estados de Mato Grosso do Sul e São Paulo (Bicudo 1972, Bueno et al. 2009).

Com relação às espécies de Nitella, Bueno et al. (2011) registraram sete novas ocorrências para os estados de Mato Grosso e Mato Grosso do Sul: N. acuminata A. Braun ex Wallman, N. axillaris A. Braun, N. cernua A. Braun, $N$. flagellifera J. Groves \& G.O. Allen, N. gollmeriana A. 
Braun, $N$. intermedia Nordstedt in T.F. Allen, $N$. mucronata (A. Braun) Miquel in H. C. van Hall e N. tenuissima (Desv.) Kützing. N. intermedia Nordstedt in T.F. Allen e N. tenuissima (Desv.) Kützing são os primeiros registros para o estado do Mato Grosso do Sul. A espécie que apresentou maior distribuição geográfica foi $N$. cernua A. Braun ocorrendo nos municípios de Campo Grande, Corumbá, Miranda e Rochedo. N. acuminata A. Braun ex Wallman foi registrada para os municípios de Bela Vista, Corumbá e Rochedo.

Em relação ao material analisado, N. acuminata A. Braun ex Wallman é a espécie que apresenta a maior distribuição geográfica em território nacional (Mato Grosso do Sul, Paraná, Rio de Janeiro, São Paulo, Rio Grande do Sul). N. acuminata A. Braun ex Wallman e N. mucronata (A. Braun) R.D. Wood foram as espécies que apresentaram a maior distribuição na área de estudo, ocorrendo em três localidades cada uma (Bela Vista, Corumbá e Rochedo e Campo Grande, Corumbá e Rochedo, respectivamente).

Com relação à espécie $N$. microcarpa A. Braun [= Nitella furcata (Roxburgh ex Bruzelius) C. Agardh emend. R.D. Wood subsp. furcata var. sieberi (A. Braun) R.D. Wood f. microcarpa (A. Braun) R.D. Wood] e suas variedades não típicas de suas respectivas espécies: $N$. microcarpa var. wrightii $\mathrm{H}$. Groves \& J. Groves [= Nitella furcata (Roxburgh ex Bruzelius) C. Agardh emend. R.D. Wood subsp. mucronata (A. Braun) R.D. Wood var. mucronata f. wrightii] e N. microcarpa var. sieberi (A. Braun) J.C. van Raam [= Nitella furcata (Roxburgh ex Bruzelius) C. Agardh emend. R.D. Wood subsp. mucronata (A. Braun) R.D. Wood var. mucronata f. wrightii], primeiro registro da espécie para o estado do Mato Grosso do Sul em Bueno \& Bicudo (1997).

$N$. tenuissima (Desvaux) Kützing e $N$. intermedia C.F.O. Nordstedt são os primeiros registros para o estado. $N$. tenuissima (Desvaux) Kützing apresentou distribuição geográfica restrita, até o momento foi registrada para os estados do Rio Grande do Sul, São Paulo, Mato Grosso do Sul, Paraná e Santa Catarina (Astorino 1983, Bueno et al. 2010, 2011, 2016) enquanto $N$. intermedia C.F.O. Nordstedt constitui o primeiro registro para o país (Bueno et al. 2011), conforme Tabela 1.

\section{Principais acervos no Brasil}

O principal acervo de material de Characeae do Brasil está no Instituto de Botânica, no Herbário "Herbário Científico do estado "Maria Eneyda P. Kauffmann Fidalgo" (SP). Esse acervo destaca-se pela organização (todo material de Characeae informatizado) e pelo número de excicatas (1.045), das quais $236(22,6 \%)$ são de material coletado no estado de São Paulo. Esses registros resultaram dos trabalhos dos especialistas e colaboradores (Dra. Rosa Maria Teixeira Bicudo e Dr. Carlos Eduardo de Mattos Bicudo) cuja meta foi inventariar a flora de Characeae do estado de São Paulo, publicada em 2004 (Picelli-Vicentim et al. 2004).
O Herbário ICN da Universidade Federal do Rio Grande do Sul foi criado em 1937 através da coleção do Prof. Dr. Alarich R. Schultz, inicialmente era uma coleção que alcançava cerca de 3.000 exemplares, atualmente faz parte do Instituto de Biociências da referida universidade, é administrado por uma Curadora (Prof ${ }^{\mathrm{a}}$. Mara Rejane Ritter) e uma Comissão Curadora formada pela primeira e mais dois professores (Lílian Eggers e João Fernando Prado) que atuam como docentes e pesquisadores. Atualmente o acervo do Herbário ICN conta com cerca de 153.000 exemplares incluídos e registrados, abrangendo Angiospermas, Gimnospermas, Pteridófitas, Briófitas, Fungos, Líquens, além das coleções especiais (como a de DNA e a carpoteca) e algas, dentre elas as Characeae do Rio Grande do Sul.

O Herbário HMS (Herbário de Mato Grosso do Sul) localizado na EMBRAPA Gado de Corte, Campo Grande, Mato Grosso do Sul foi fundado em 2000, pelos pesquisadores Vali Joana Pott e Arnildo Pott, atualmente possui aproximadamente 13.000 exsicatas, os espécimes pertencentes a este acervo representam a grande biodiversidade da flora do estado, oriundos do Pantanal, do Cerrado e do Chaco brasileiro.

O Herbário CGMS da Universidade Federal do Mato Grosso do Sul foi fundado em 1989, atualmente possui aproximadamente 27.000 exsicatas, os espécimes pertencentes a este acervo representam a grande biodiversidade da flora do estado, oriundos do Pantanal, do Cerrado e do Chaco brasileiro. O Herbário CGMS é administrado por uma curadora (Dra. Ângela Lúcia Bagnatori Sartori \& Vali Joana Pott, Especialistas) especialistas em leguminosas, plantas aquáticas do pantanal e flora de veredas. Em função dos projetos em áreas úmidas muitas Characeae foram coletadas e encontramse depositadas e identificadas naquele Herbário.

O Herbário CPAP da Embrapa foi criado em 1984 pelos pesquisadores Vali Joana Pott e Arnildo Pott, possui aproximadamente 19.000 exsicatas de plantas das diversas sub-regiões do Pantanal, da Bacia do Alto Paraguai e outras regiões. Tais coleções permitiram a elaboração dos livros "Plantas do Pantanal" e "Plantas Aquáticas do Pantanal" e a coleta e identificação das Characeae do Pantanal dos estados do Mato Grosso e Mato Grosso do Sul.

O Herbário UNOP e UNOP-Algae da Universidade Estadual do Oeste do Paraná foi criado em 1996 pelo professor Dr. Bartolomeu Tavares, atualmente é coordendo pelas doutoras Lívia Temponi, Shirley Silva e Norma Bueno. Possui cerca de 13.000 amostras e foi inserido no Index Herbariorum, reunindo principalmente representantes de Charophyta, Chlorophyta e Bacillariophyta, além das Embriófitas. Abriga as coleções de Characeae recebidas pelo processo de permuta para identificação de vários estados brasileiros. Recentemente graças ao trabalho de coleta do Prof. Dr. José Alves de Siqueira Universidade Federal do Vale do São Francisco (UNIVASF) Curador Herbário Vale do São Francisco (HVASF) do Centro de Referência para a Recuperação de Áreas Degradadas (CRAD), foi possível 
a identificação de sete espécies de Chara e uma espécie de Nitella (Siqueira Filho 2012). Em 2013, graças ao Programa de Pós-Graduação em Conservação e manejo de Recursos Naturais, da Universidade Estadual do Oeste do Paraná, foi aprovado pela FINEP o Subprojeto: Infraestrutura multiusuária de pesquisa e Pós-Graduação em Conservação e Manejo de Recursos Naturais (Edital MCTI/FINEP/CTINFRA 01/2013), sob a coordenação da Dra. Bueno, para a construção de um prédio com uma área total de 1.491,52 $\mathrm{m}^{2}$ que abrigará 18 laboratórios de pesquisa dentre eles os Herbários de Fanerógamas e Criptógamas.

\section{MATERIAL E MÉTODOS}

A compilação dos dados do presente trabalho está baseada em artigos científicos em revistas indexadas, ou ainda mediante registros de espécies em coleções brasileiras e estrangeiras: Braun \& Nordstedt (1883), Hoehne (1914, 1923, 1936), Hoehne \& Kuhlmann (1951), Bicudo (1968a, 1968b, 1972, 1974), Bicudo \& Yamaoka (1978), Pott et al. (1992), Bueno et al. (1996), Bueno et al. (2009) e Bueno et al. (2011).

As coleções acessadas foram: Herbário SP (Instituto de Botânica, São Paulo), Herbário HMS (Herbário de Mato Grosso do Sul) localizado na EMBRAPA Gado de Corte, Campo Grande, Mato Grosso do Sul, Herbário CGMS (Herbário da Fundação Universidade Federal de Mato
Grosso do Sul) localizado na UFMS, Campo Grande, Mato Grosso do Sul, Herbário CPAP (Centro de Pesquisa Agropecuária do Pantanal) localizado na EMBRAPA Pantanal, Corumbá, Mato Grosso do Sul e Herbário UNOP (Universidade Estadual do Oeste do Paraná), localizado na Unioeste, Cascavel, Paraná. Os registros dos herbários continham informações de localização, porém careciam de informações a respeito das características físicas e químicas dos locais de amostragem.

A classificação seguiu Krause (1997) e as seguintes literaturas foram consultadas para as identificações e discussões dos espécimes: Bicudo (1974, 1977, 1979), Bueno et al. (1996), Bueno et al. (2009), Groves \& Groves (1911), Moore (1986), Picelli-Vicentim et al. (2004), Proctor et al. (1971), Vieira-Júnior et al. (2003), Wood \& Imahori $(1964,1965)$, Bueno et al. (2011). O preparo das lâminas para estudo e identificação taxonômica dos materiais seguiu método descrito em Bicudo (1974) e Bicudo \& Menezes (2006).

\section{RESULTADOS E DISCUSSÃO}

Foram identificadas 16 espécies dos gêneros Chara L. e Nitella C. Agardh (Characeae, Streptophyta) para o

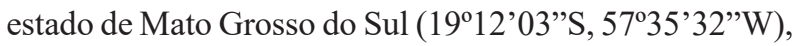
Brasil. A distribuição geográfica dos táxons no estado de Mato Grosso do Sul e Brasil encontram-se no Quadro 1.

Quadro 1. Distribuição geográfica das Characeae no Brazil e no Estado de Mato Grosso do Sul (MS).

\begin{tabular}{|c|c|c|}
\hline Charophyceae & Distribuição no Brasil & Distribuição no MS \\
\hline Chara guairensis R. Bicudo & $\begin{array}{l}\text { Paraná (Bicudo 1972, 1974); São Paulo (Picelli- } \\
\text { Vicentim et al. 2004, Vieira-Júnior } \text { et al. 2003); Rio } \\
\text { Grande do Sul (Prado 2003) }\end{array}$ & $\begin{array}{l}\text { Aquidauna [Pantanal, sub-região de } \\
\text { Aquidauana], Bodoquena, Bonito, Campo } \\
\text { Grande, Corumbá [Pantanal, sub-região do } \\
\text { Abobral, Nabileque e Paraguai], Inocência, } \\
\text { Miranda, Porto Murtinho (Bueno et al. } \\
\text { 1996, Bueno et al. 2009) }\end{array}$ \\
\hline C.hydropitys Reichenbach & $\begin{array}{l}\text { C. hydropitys Reichenbach [=C. fibrosa C. Agardh } \\
\text { ex Bruzelius emend. R.D Wood var. hydropitys } \\
\text { (Reichenbach) R.D. Wood emend. R.D. Wood f. } \\
\text { hydropitys]. Bahia (Braun \& Nordstedt 1883; Bicudo } \\
\text { 1972); Maranhão (Bicudo 1972); Rio Grande do Sul } \\
\text { (Astorino 1983; Torgan et al. 2007; Prado 2003). }\end{array}$ & $\begin{array}{l}\text { Corumbá [Pantanal, sub-região do Abobral, } \\
\text { sub-região do Nabileque e sub-região da } \\
\text { Nhecolândia] (Bicudo 1972; Bueno et al. } \\
\text { 1996); Bonito, Campo Grande e Porto } \\
\text { Murtinho, (Bueno et al. 2009) }\end{array}$ \\
\hline C. kenoyeri Howe & $\begin{array}{l}\text { Espírito Santo (Bicudo 1972, 1974; Proctor et al. } \\
\text { 1971); Goiás (Wood \& Imahori 1965); Minas Gerais } \\
\text { (Bicudo 1972, 1974) }\end{array}$ & Bonito, Campo Grande (Bueno et al. 2009) \\
\hline C. martiana Wallman & $\begin{array}{c}\text { Goiás e Piauí (Bicudo 1972, 1974); São Paulo (Bicudo } \\
\text { 1972, 1974; Picelli-Vicentim et al. 2004; Necchi-Júnior } \\
\text { et al. 1995; Necchi-Júnior et al. 1997; Necchi-Júnior } \\
\text { et al. 2000; Vieira-Júnior 2001); Rio Grande do Sul } \\
\text { (Prado 2003). }\end{array}$ & $\begin{array}{c}\text { Corumbá, Bonito, Ladário (Bueno et al. } \\
\text { 1996, Bueno et al. 2009) }\end{array}$ \\
\hline C. rusbyana Howe & $\begin{array}{l}\text { Mato Grosso (Bicudo 1972, 1974); Minas Gerais, } \\
\text { Pernambuco, Piauí, Santa Catarina (Bicudo 1972, 1974); São } \\
\text { Paulo (Bicudo 1972, 1974; Picelli-Vicentim 1990, Picelli- } \\
\text { Vicentim et al. 2004); Rio Grande do Sul (Prado 2003). }\end{array}$ & $\begin{array}{l}\text { Aquidauana, Bodoquena, Bonito, Corumbá } \\
\text { [Pantanal, sub-região do Abobral, Nabileque } \\
\text { e Nhecolândia], Ladário, Miranda [Pantanal, } \\
\text { sub-região de Miranda, Porto Murtinho } \\
\text { (Bueno et al. 1996, Bueno et al. 2009) }\end{array}$ \\
\hline
\end{tabular}


Quadro 1. Cont.

\begin{tabular}{|c|c|c|}
\hline Charophyceae & Distribuição no Brasil & Distribuição no MS \\
\hline $\begin{array}{l}\text { C.socotrensis f. socotrensis } \\
\text { (Nordstedt ex Kuhn) R.D.Wood } \\
\text { 1962: } 13\end{array}$ & São Paulo (Bicudo 1972) & Bonito, Porto Murtinho (Bueno et al. 2009) \\
\hline $\begin{array}{l}\text { Nitella acuminata A. Braun ex } \\
\text { Wallm }\end{array}$ & $\begin{array}{l}\text { Paraná (Thomaz et al. 2003); Rio de Janeiro (Bicudo } \\
\text { 1969, Bicudo \& Yamaoka 1978); São Paulo (Picelli- } \\
\text { Vicentim 1990, Bicudo 1969, Bicudo \& Yamaoka 1978, } \\
\text { Picelli-Vicentim \& Bicudo 1993, Picelli-Vicentim et al. } \\
\text { 2004); Rio Grande do Sul (Prado 2003) }\end{array}$ & $\begin{array}{c}\text { Bela Vista, Corumbá [Pantanal, sub-região } \\
\text { do Abobral], Rochedo (Bueno \& Bicudo } \\
\text { 1997, Bueno et al. 2011) }\end{array}$ \\
\hline N. axillaris A. Braun & $\begin{array}{l}\text { N. axillaris A. Braun [=N. translucens (Persoon) C. } \\
\text { Agardh emend. R.D. Wood subsp. translucens var. } \\
\text { axillaris (A. Braun) R.D. Wood f. axillaris]. } \\
\text { Pernambuco (Wood \& Imahori 1965); São Paulo (Picelli- } \\
\text { Vicentim 1990, Picelli-Vicentim \& Bicudo 1993, Picelli- } \\
\text { Vicentim et al. 2004); Rio Grande do Sul (Prado 2003) }\end{array}$ & $\begin{array}{l}\text { Corumbá [Pantanal, sub-região Abobral } \\
\text { e Nabileque], Porto Murtinho, (Bueno \& } \\
\text { Bicudo 1997, Bueno et al. 2011) }\end{array}$ \\
\hline N. cernua A. Braun & $\begin{array}{l}\text { Fernando de Noronha (Groves \& Groves 1911); Minas } \\
\text { Gerais (Wood \& Imahori 1965); São Paulo (Wood \& } \\
\text { Imahori 1965, Bicudo \& Yamaoka 1978, Picelli-Vicentim } \\
\text { 1990, Necchi-Júnior et al. 2000, Picelli-Vicentim \& Bicudo } \\
\text { 1993, Vieira-Júnior et al. 2002, Picelli-Vicentim et al. 2004) }\end{array}$ & $\begin{array}{l}\text { Corumbá [Pantanal, sub-região Abobral e } \\
\text { Nabileque](Bueno \& Bicudo 1997) }\end{array}$ \\
\hline $\begin{array}{l}\text { N. flagellifera J. Groves \& G.O. } \\
\text { Allen }\end{array}$ & $\begin{array}{l}\text { N. flagellifera J. Groves \& G.O. Allen: [= N. furcata } \\
\text { (Roxburgh ex Bruzelius) C. Agardh emend. R.D. Wood } \\
\text { subsp. flagellifera (J.Groves \& G.O. Allen) R.D. Wood]. } \\
\text { São Paulo (Picelli-Vicentim 1990, Vieira-Júnior } \text { et al. 2002, } \\
\text { Picelli-Vicentim et al. 2004); Rio Grande do Sul (Prado }\end{array}$ & $\begin{array}{c}\text { Corumbá [Pantanal, sub-região do Abobral], } \\
\text { Miranda (Bueno \& Bicudo 1997, Bueno et } \\
\text { al. 2011) }\end{array}$ \\
\hline N. gollmeriana A. Braun & $\begin{array}{c}\text { 2003) } \\
\text { São Paulo (Bicudo 1969, Bicudo \& Yamaoka 1978, } \\
\text { Picelli-Vicentim 1990, Branco \& Necchi-Júnior 1996, } \\
\text { Necchi-Júnior } \text { et al. 2000, Vieira-Júnior } \text { et al. 2002, } \\
\text { Picelli-Vicentim et al. 2004); Rio Grande do Sul (Prado } \\
\text { 2003) }\end{array}$ & $\begin{array}{l}\text { Corumbá [Pantanal, sub-região do Nabileque } \\
\text { e Nhecolândia] (Bueno \& Bicudo 1997) }\end{array}$ \\
\hline $\begin{array}{l}N \text {. intermedia C.F.O. Nordstedt. in } \\
\text { T.F. Allen }\end{array}$ & & Corumbá (Bueno et al. 2011) \\
\hline N. microcarpa A. Braun & $\begin{array}{l}\text { N. microcarpa Braun [ }=N \text {. furcata (Roxburgh ex Bruzelius) } \\
\text { C. Agardh emend. R.D. Wood subsp. furcata var. sieberi } \\
\text { (A. Braun) R.D. Wood f. microcarpa (A. Braun) R.D. } \\
\text { Wood] São Paulo (Picelli-Vicentim 1990, Necchi-Júnior et } \\
\text { al. 2000, Vieira-Júnior 2002, Picelli-Vicentim et al. 2004) }\end{array}$ & $\begin{array}{l}\text { Aquidauana (Bueno \& Bicudo 1997, Bueno } \\
\text { et al. 2011) }\end{array}$ \\
\hline $\begin{array}{l}\text { N. microcarpa var. sieberi (A. } \\
\text { Braun) J.C. van Raam }\end{array}$ & $\begin{array}{l}\text { N. microcarpa var. sieberi (A.Braun) J.C. van Raam 2010: } \\
\text { [= N. furcata (Roxburgh ex Bruzelius) C. Agardh emend. } \\
\text { R.D. Wood subsp. mucronata (A. Braun) R.D. Wood var. } \\
\text { mucronata f. wrightii]. São Paulo (Picelli-Vicentim 1990, } \\
\text { Necchi-Júnior et al. 2000, Vieira-Júnior et al. 2002, Picelli- } \\
\text { Vicentim et al. 2004), Rio Grande do Sul (Astorino 1983) }\end{array}$ & $\begin{array}{l}\text { Corumbá (Bueno \& Bicudo 1997, Bueno et } \\
\text { al. 2011) }\end{array}$ \\
\hline $\begin{array}{l}\text { N. microcarpa var. wrightii } \mathrm{H} . \\
\text { Groves \& J. Groves }\end{array}$ & $\begin{array}{l}\text { N. microcarpa var. wrightii H. Groves \& J. Groves [= } \\
\text { N. furcata (Roxburgh ex Bruzelius) C. Agardh emend. } \\
\text { R.D. Wood subsp. mucronata (A. Braun) R.D. Wood } \\
\text { var. mucronata f. wrightii]. São Paulo (Picelli-Vicentim } \\
\text { 1990, Necchi-Júnior } \text { et al. 2000, Vieira-Júnior et al. 2002, } \\
\text { Picelli-Vicentim et al. 2004) }\end{array}$ & $\begin{array}{l}\text { Corumbá (Bueno \& Bicudo 1997, Bueno et } \\
\text { al. 2011) }\end{array}$ \\
\hline $\begin{array}{l}\text { N. mucronata (A. Braun) F. } \\
\text { Miquel in H.C. Hall }\end{array}$ & $\begin{array}{l}\text { N. mucronata (A. Braun) F. Miquel in H.C. Hall: [= } \\
\text { N. furcata (Roxburgh ex Bruzelius) C. Agardh emend. } \\
\text { R.D. Wood subsp. mucronata (A. Braun) R.D. Wood } \\
\text { var. mucronata f. mucronata]. Ceará (Wood \& Imahori } \\
\text { 1965); São Paulo (Picelli-Vicentim 1990, Necchi- } \\
\text { Júnior et al. 2000, Vieira-Júnior et al. 2002, Picelli- } \\
\text { Vicentim et al. 2004, Branco \& Necchi-Júnior 1998) }\end{array}$ & $\begin{array}{l}\text { Campo Grande, Corumbá [Pantanal sub- } \\
\text { região Abobral, sub-região do Nabileque, } \\
\text { sub-região do Paraguai e sub-região de } \\
\text { Miranda], Rochedo, (Bueno \& Bicudo } \\
\text { 1997, Bueno et al. 2011) }\end{array}$ \\
\hline
\end{tabular}


Quadro 1. Cont.

\begin{tabular}{|c|c|c|}
\hline Charophyceae & Distribuição no Brasil & Distribuição no MS \\
\hline N. subglomerata A. Braun & $\begin{array}{l}\text { Mato Grosso (Braun \& Nordstedt 1883, Bicudo } \\
\text { \& Yamaoka 1978), Minas Gerais (Braun 1883 \& } \\
\text { Nordstedt, Bicudo 1969, Bicudo \& Yamaoka 1978), } \\
\text { Paraná (Bicudo \& Yamaoka 1978), Rio Grande do } \\
\text { Sul (Astorino 1983, Prado 2003), São Paulo (Braun } \\
\text { \& Nordstedt 1883, Wood \& Imahori 1965, Bicudo \& } \\
\text { Yamaoka 1978, Picelli-Vicentim 1990, Picelli-Vicentim } \\
\text { \& Bicudo 1993, Necchi-Júnior et al. 2000, Picelli- } \\
\text { Vicentim et al. 2004, Vieira-Júnior et al. 2002). }\end{array}$ & $\begin{array}{l}\text { Brasilândia (Bicudo \& Yamaoka 1978), } \\
\text { Corumbá [Pantanal sub-região do Abobral e } \\
\text { sub-região do Nabileque] (Bueno \& Bicudo } \\
\text { 1997, Bueno et al. 2011) }\end{array}$ \\
\hline N. tenuissima (Desvaux) Kützing & $\begin{array}{l}\text { Rio Grande do Sul (Astorino 1983, Vieira-Júnior et al. 2002, } \\
\text { Prado 2003); São Paulo (Picelli-Vicentim et al. 2004). }\end{array}$ & Porto Murtinho (Bueno et al. 2011) \\
\hline
\end{tabular}

\section{Perspectivas de Pesquisa para o Grupo nos próximos 10 anos}

De acordo com Bicudo \& Bueno (2011) duas possibilidades de avanço na pesquisa em Characeae podem ser consideradas, que seguem as duas linhas da taxonomia: a linha $\alpha$ (exclusivamente morfológica) e a linha $\omega$ (subsidiada por outros campos da biologia ou mesmo por outras ciências).

Seguindo a linha $\alpha$ não como perspectiva, mas como ação em andamento, consta a organização da monografia das Characeae do projeto "Flora Neotropica", de autoria de Norma Catarina Bueno, Carlos Eduardo de Mattos Bicudo, João Fernando Prado e Thamis Meurer, além de uma nova parceria que esta sendo estabelecida para a realização da Flora da Bahia, de autoria de Carlos Walace do Nascimento Moura, Norma Catarina Bueno, Carlos Eduardo de Mattos Bicudo e João Fernando Prado. Com relação à linha $\omega$ a perspectiva em curto prazo é a formação de mais especialistas em taxonomia de Characeae, atualmente Norma Catarina Bueno, João Fernando Prado e Thamis Meurer, trabalhando no sul do país. Em médio prazo, a perspectiva é o aprimoramento da taxonomia clássica das Characeae pelo uso de técnicas da microscopia eletrônica de varredura para uma melhor definição da decoração da parede dos oósporos (caráter importante na taxonomia providenciada em nível infragenérico); também, a avaliação da taxonomia clássica (morfologista) das Characeae pelo uso (1) de técnicas de biologia molecular e (2) de biossistemática, para uma melhor definição da circunscrição de espécies, variedades e formas taxonômicas.

\section{AGRADECIMENTOS}

Carlos Eduardo de Mattos Bicudo e Norma Catarina Bueno agradecem ao Conselho Nacional de Desenvolvimento Científico e Tecnológico, por Bolsa de Produtividade e à Fundação Parque Tecnológico Itaipu, pela bolsa nível Mestrado à segunda autora.

\section{REFERÊNCIAS}

Astorino, H.A.B. 1983. Charophyceae do estado do Rio Grande do Sul: uma contribuição ao seu inventário. Dissertação 108 f., Universidade Estadual Paulista "Júlio de Mesquita Filho", Rio Claro.

Bicudo, R.M.T. 1968a. An annotated list of Charophyceae already cited for Brazil. Rickia 3:221-238

1968b. Index to the Brazilian cryptogamic literature, 1: a bibliography of the Brazilian Charophyceae. Rickia 3:239-245.

1969. Brazilian Characeae of the herbarium of the Instituto de Botânica, São Paulo. Nova Hedwigia 17:1-17. 1972. O gênero Chara (Charophyceae) no Brasil. Tese $229 \mathrm{f}$. Universidade de São Paulo, São Paulo. 1974. O gênero Chara (Charophyceae) no Brasil, 1: Subseção Willdenowia R.D. Wood. Rickia 6:127-189.

1976. Chara linharensis, uma nova espécie de Characeae do sudeste do Brasil. Ciência e Cultura 28:1314-1318.

1977. O gênero Chara (Charophyceae) no Brasil, 2: Seção Chara. In Anais do XXVI Congresso Nacional de Botânica. Rio de Janeiro, p.23-32.

. 1979. O gênero Chara (Charophyceae) no Brasil, 3: Seção Charopsis (Kützing emend. Rupr., Leonh.) R.D. Wood. Rickia 8:17-26.

Bicudo, C.E.M. \& Bueno, N.C. 2011. "Check List" das Charophyceae do Estado de São Paulo. Biota Neotropica, 11 (1a). Disponível em: http://www.biotaneotropica.org.br/v11n1a/pt/abstract?inventory+b n0081101a2011. Acessado em 20.01.2013.

Bicudo, C.E.M. \& Menezes, M. 2006. Gêneros de algas de águas continentais do Brasil. Editora RiMa, São Paulo. 489 p.

Bicudo, R.M.T. \& Yamaoka, D.M. 1978. O gênero Nitella (Charophyceae) no Brasil, 1: subgênero Nitella. Acta Biologica Paranaense 7:77-98.

Branco, C.C.Z. \& Necchi-JÚnior, O. 1996. Survey of stream macroalgae of eastern Atlantic Rainforest of São Paulo State, southeastern Brazil. Algological Studies 80:35-57.

Branco, C.C.Z. \& Necchi-Júnior, O. 1998. Distribution of stream macroalgae in three tropical drainage basins of southeastern Brazil. Archiv fur Hydrobiologie 142:241-256.

Braun, A. \& Nordstedt, C.F.O. 1883. Fragmente einer Monographie der Characeen: nach der hinterlassenen Manuscripten. A. Braun's herausgegeben von Dr. Otto Nordstedt. Abhandlungen der Königinen Akademie der Wissenchaftlichen Berlin. p.1-211.

Bueno, N.C., Bicudo, C.E.M., Picelli-Vicentim, M.M. \& ISHII, I.H. 1996. Characeae (Charophyceae) do pantanal de Mato Grosso do Sul, Brasil: Chara. Hoehnea 23(2):21-31.

Bueno, N.C. \& Bicudo, C.E.M. 1997. Characeae (Charophyceae) do Pantanal de Mato Grosso do Sul, Brasil: Nitella. Hoehnea 24:29-55.

Bueno, N.C., Bicudo, C.E.M., Biolo, S. \& Meurer, T. 2009. Levantamento florístico das Characeae (Chlorophyta) de Mato Grosso e Mato Grosso do Sul, Brasil: Chara. Revista Brasileira de Botânica 32:759-774. 
Bueno, N.C., Meurer, T., Biolo, S. \& Bicudo, C.E.M. 2011. Novos registros de Nitella (Chlorophyta, Characeae) para as regiões dos Estados de Mato Grosso e Mato Grosso do Sul. Hoehnea 38(3):385396.

Bueno, N.C., Meurer, T., Biolo, S. \& Bicudo, C.E.M. 2011. Novos registros de Nitella (Chlorophyta, Characeae) para as regiões dos Estados de Mato Grosso e Mato Grosso do Sul. Hoehnea 38(3):385-396.

Bueno, N.C., Prado, J.F., Meurer, T., Biolo, S. \& Bicudo, C.E.M. 2016. Nitella (Streptophyta, Characeae) from southern Brazil. Iheringia. Serie Botânica 71(2):132- 154.

Groves, H. \& Groves, J. 1911. Characeae. In Urban, I.(ed). Symbolae Antillanae, seu Fundamenta. Florae Indiae Occidentalis 7(1): 30-44.

Hoehne, F.C. 1914. Expedição Scientifica Roosevelt-Rondon. Relatório apresentado ao Snr. Coronel de Engenharia Candido Marianno da Silva Rondon, Chefe da Comissão Brasileira. Rio de Janeiro, editora não mencionada. p.1-81 (anexo 2).

1923. Phytophysionomia do Estado de Matto-Grosso e ligeiras notas a respeito da composição e distribuição da sua flora: estudo preliminar. São Paulo, Companhia Melhoramentos de São Paulo. p.1-104.

1936. O Grande Pantanal de Mato-Grosso. Boletim de Agricultura 37:443-470

Hoehne, F.C. \& Kuhlmann, J.G. 1951. Índice bibliográfico e numérico das plantas colhidas pela Comissão Rondon ou Comissão de Linhas Telegráficas, Estratégicas de Mato-Grosso ao Amazonas, de 1908 até 1923. Secretaria de Agricultura, São Paulo,.1-400.

Krause, W. 1997. Charales (Charophyceae). Subwasserflora von Mitteleuropa. Vol. 18. eds. H. Ettl, G. Gärtner, H. Heyning, and D. Molenhauser. Sttutgart: Gustav Fischer Verlag, Jena. 202 p.

Moore, J.A. 1986. Charophytes of Great Britain and Ireland. Botanical Society of the British Isles, London.

Necchi-Júnior, O., Branco, C.C.Z. \& Branco, L.H.Z. 2000. Distribution of stream macroalgae in São Paulo State, southeastern Brazil. Algological Studies 97:43-57.

Necchi-Júnior, O., Branco, C.C.Z., Simão, R.C.G. \& Branco, L.H.Z. 1995. Distribution of stream macroalgae in the northwest region of São Paulo State, southeastern Brazil. Hydrobiologia 299:291-230.

Necchi-Júnior, O., Pascoaloto, D. \& Branco, L.H.Z. 1997. Stream macroalgal flora from the northwest region of São Paulo state, southeastern Brazil. Algological Studies 84:91-112.

Picelli-Vicentim, M.M. 1990. Characeae do Estado de São Paulo: inventário sistemático. Tese 256 f. Universidade Estadual Paulista, Rio Claro.
Picelli-Vincentim, M.M. \& Bicudo, C.E.M. 1993. Criptógamos do Parque estadual das Fontes do Ipiranga, São Paulo, SP. Algas, 4: Charophyceae. Hoehnea 20:9-22.

Picelli-Vicentim, M.M., Bicudo, C.E.M. \& Bueno, N.C. 2004. Flora ficológica do Estado de São Paulo, 5: Charophyceae. São Paulo: RiMa Editora. 124 p.

Pott, V.J., Bueno, N.C. \& Silva, M.P. 1992. Levantamentoflorístico e fitossociológico de macrófitas aquáticas em lagoas da Fazenda Leque, Pantanal, MS. In Anais do VIII Congresso da Sociedade Botânica de São Paulo. Sociedade Botânica de São Paulo, Campinas, p.91-99.

Prado, J.F. 2003. Characeae do Rio Grande do Sul, Brasil. Tese, 233f., Universidade Federal do Rio Grande do Sul, Porto Alegre.

Proctor, V.W., Griffin III, D.G. \& Hotchkiss, A.T. 1971. A sinopsis of the genus Chara, series Gymnobasalia (subsection Willdenowia R.D. Wood). American Journal of Botany 58:894-901.

Siqueira Filho, J.A. 2012. Flora of the Caatingas of the São Francisco River: natural history and conservation (J. A. de Siqueira Filho, org.), Andrea Jakobsson, Rio de Janeiro. 552p.

Thomaz, S.M., Souza, D.C. \& Bini, L.M. 2003. Species richness and beta diversity of aquatic macrophytes in a large sub-tropical reservoir (Itaipu Reservoir, Brazil): the influence of limnology and morphometry. Hydrobiologia 505:119-128.

Torgan, L.C., Alves, S.M., Werner, V.R., Rosa, Z.M., Cardoso, L.S., Rodrigues, S.C., Santos, C.B., Palma, C., Fortuna, J., Bicca, A.B. \& Weber, A.S. 2007. Ficoflora. In Biodiversidade. Regiões da Lagoa do Casamento e dos Butiazais de Tapes, Planície Costeira do Rio Grande do Sul (Becker, F.G., Ramos, R.A. \& Moura L.A., org.). Ministério do Meio Ambiente, Brasília. p.112-127.

Vieira-Júnior, J. 2001. Characeae (Chlorophyta) de ecossistemas lóticos do Estado de São Paulo: levantamento florístico, dinâmica espaçotemporal, distribuição geográfica e análise ecofsiológica. Dissertação 268 f. Universidade Estadual Paulista, Rio Claro.

Vieira-Júnior, J., Necchi-Júnior, O., Branco, C.C.Z. \& Branco, L.H.Z. 2002. Characeae (Chlorophyta) em ecossistemas lóticos do Estado de São Paulo, Brasil: gênero Nitella. Hoehnea 29:249-266.

2003. Characeae (Chlorophyta) em ecossistemas lóticos do Estado de São Paulo, Brasil: gênero Chara e distribuição ecológica. Hoehnea 30:53-70.

Wood, R.D. \& Imahori, K. 1964. A revision of the Characeae, 2: iconograph of the Characeae. J. Cramer, Weinhen. 395p.

. 1965. A revision of the Characeae, 1: monograph of the Characeae. J. Cramer, Weinhen. 994p. 